Dept. of Parasitology,

Fac. Vet. Medicine, Kafr Elsheikh University,

\title{
EPIDEMIOLOGICAL STUDIES ON HARD TICKS AND TICK BORNE PARASITES, IN SHALATIN CITY, RED SEA GOVERNORATE, EGYPT
}

(With 9 Tables and 25 Figures)

\author{
By \\ M.A. EL-SEIFY; O.M. MAHRAN* \\ and A.M.I. ABD EL AAL**
}

Animal Health Research Institute (ARC), Dokki, Giza, Egypt (*Shalatin,

**Matrouh Branches)

(Received at 9/6/2011)

\section{دراسات وبائية عن القراد الجامد والطفيليات المنقولة به بمدينة شلاتيذ، الإية، مدرية

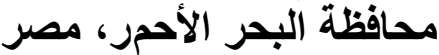

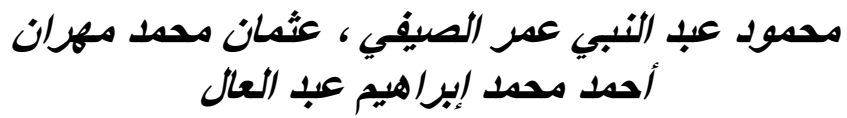

تم فحص عدد 551 من الإبل و 225 من الأغنام و 106 من الماعز وذللك لمعرفة معدل

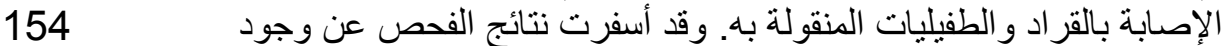

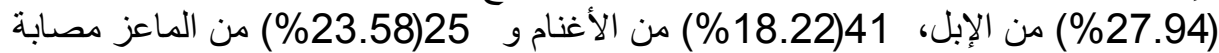

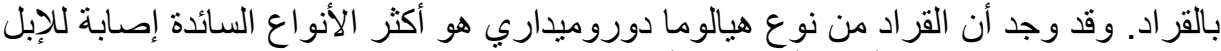

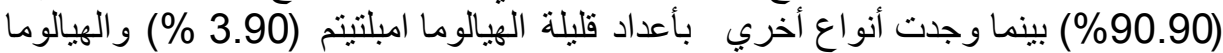

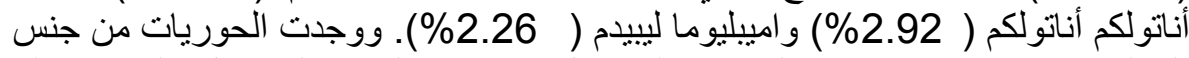

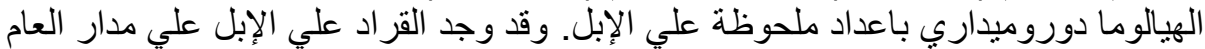

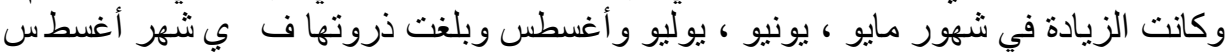

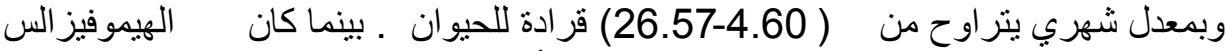

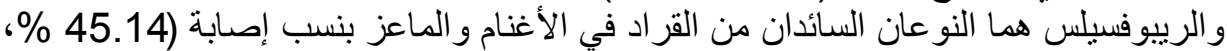

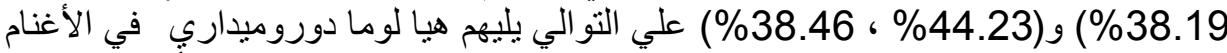

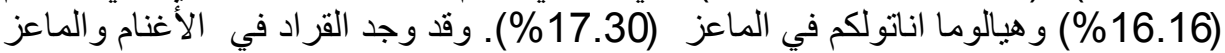

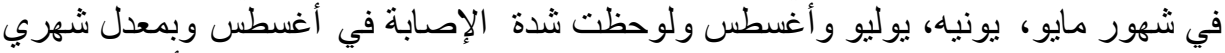

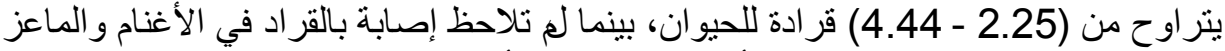

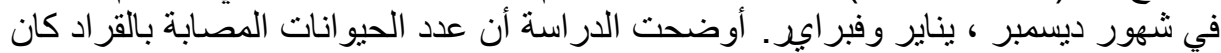

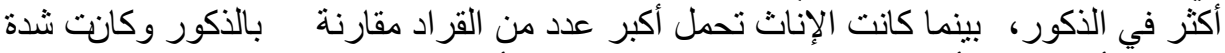

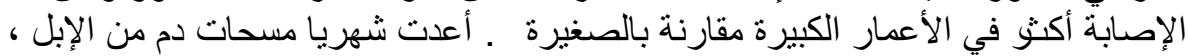




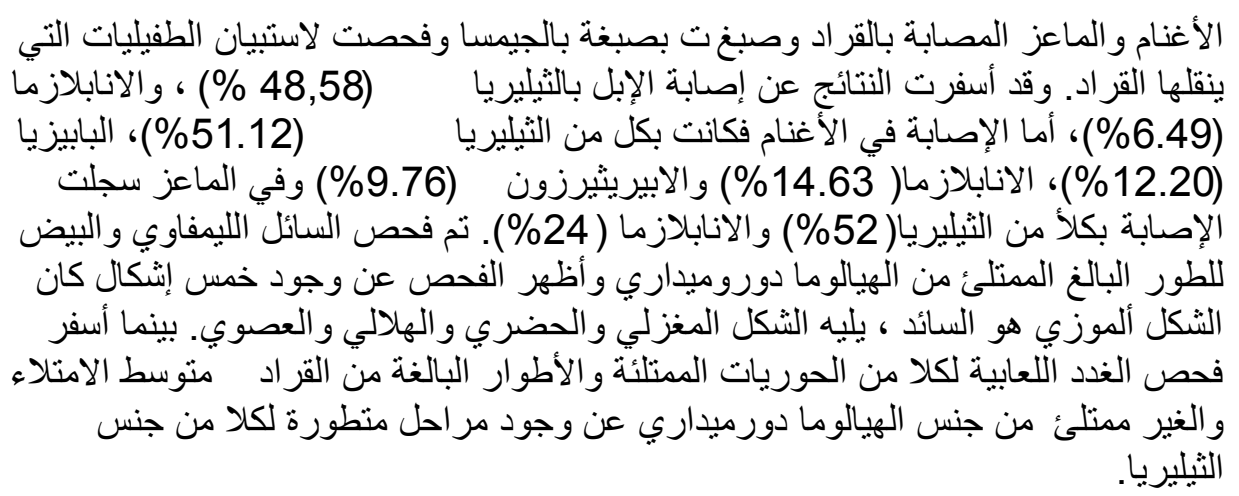

\section{SUMMARY}

A total of 551 camels, 225 sheep, and 106 goats were examined for the prevalence of tick infestation and tick borne haemoparasites. Tick infestation was detected in $154(27.94 \%)$ in camels, 41 (18.22\%) in sheep and $25(23.58 \%)$ in goats. Hyalomma dromedarii was found to be the predominant tick species $(90.90 \%)$ infesting camels. Other tick species found in low numbers were (Hyalomma impeltatum (3.90\%), Hyalomma anatolicum anatolicum (2.93\%) and Amblyomma lepidum (2.26\%). Nymphs of the genus Hyalomma were collected in significant numbers in camels. Ticks were found on camels throughout the year and increased in numbers during May, Jun, July and August with a peak in August and a mean monthly total of (4.60-26.57) ticks per animal. Rhipcephalus sp., and Haemophysalis $s p$., found to be the predominant tick species $(45.14 \% \& 38.19 \%)$ and $(44.23 \% \& 38.46 \%)$ among sheep and goats respectively followed by Hyalomma dromedarii in sheep (16.66\%) and $H$. anatolicum anatolicum $(17.30 \%)$ ) in goats. Ticks were found on sheep and goats during May, Jun, July and August with a peak in August and a mean monthly total of (2.254.44) ticks per animal. No sheep and goats were found infested with ticks from December - February. Tick infestation was more prevalent in male while female animals harbored more ticks infestation than males, higher numbers of ticks per animals were found in older animals as compared younger ones. Giemsa-stained blood smears prepared monthly from tick infested camels, sheep and goats revealed the presence of Theileria camelensis (48.58\%), and Anaplasma marginale (6.49\%) in camels. Theileria ovis. (51.21\%), Babesia sp., (12.20\%), Anaplasma marginale (14.63\%) and Eperythrozoon sp., (9.76\%) in sheep and Theileria ovis (52\%) and Anaplasma marginale (24\%) in goats. Examination of the haemolymph and egg smears of the obtained engorged adult female ticks revealed presence 
of five forms, the banana forms were the highest, followed by the spindle, club, crescentic and rod. While examination of salivary glands of engorged nymphs and moderate feed and unfed adult H.dromedarii ticks showed the developmental stages of Theileria.

Key words: Hard ticks, tick borne parasites, camel, sheep, goats.

\section{INTRODUCTION}

Hard ticks are very important pests of wildlife, domestic animals, and humans. Their irritating bites cause extreme pain and economic losses in domestic animals and a great deal of discomfort to people who work or play in tick infested area. In addition, ticks transmit several diseasecausing organisms to animals and humans (Caeiro, 1999). Tick infestation has been reported to affect appetite, body condition, blood composition and respiratory rate of animals (Gerrit, 1986). In temperate and tropical countries, ticks surpass all other arthropods in the number and variety of diseases they transmit to animals, (Friedhoff, 1997). The tick-borne diseases of livestock constitute a complex of several diseases whose etiological agents may be protozoal, rickettsial and bacterial, (Caeiro, 1999). Tick-borne diseases are present throughout the world, but are most numerous and exert their greatest impact in the tropical and subtropical regions (Gerrit, 1986). Diseases transmit by ticks have been a major constraint to the improvement of livestock industries, particularly in the developing countries for the past 100 years. In many countries, they are the major health impediments to efficient livestock production. On a global basis, the economic losses due to all caused by tick borne diseases is staggering. (Barnett, 1974a and 1974b). Although over 60 tick-borne agents may be pathogenic to livestock throughout the world, relatively few are recognized to be of economic significance. In addition to being efficient vectors of diseases agents, it has been estimated that there is a loss of 1-3 $\mathrm{ml}$ of blood for every tick completing its life cycle on an animal (Higgins, 1984). Furthermore, tick infestations cause irritation, damage hides, and predispose animals to bacterial and fungal infections, as well as screw-worm attack, in the wounds left by tick bites (Friedhoff, 1997). Recent studies indicate that the total annual loss caused by the tick amounts to about $5 \$$ per head of animals, or 4 percent of the gross value of animal slaughtered (Norval et al., 1992). The vast majority of recent studies of tick prevalence on livestock have been carried out in Egypt by 
Mazyad and Khalaf (2002) in El Arich city, El Hassana and El-Refaii and Wahba (2003) in Cairo abattoir. Other countries in Mediterranean regions, indicating a perceived greater importance of these parasites. In the southern part of the Egyptian desert Shalatin area no data about the epidemiology of ticks and tick -borne parasites. Thus the present study has been formulated to attain the following surveying the ticks infesting livestock in this area, determination of the seasonal pattern of tick infestation, observation of the prevalence of tick infestation with respect to host (age, species and gender), and determine the blood parasites that may be transmitted by ticks.

\section{MATERIALS and METHODS}

\section{Study area}

This study was carried out in Shalatin city which represents the southern part of the Egyptian desert. Climatically, this area is further categorized as semi- arid area characterized by climate with long dry hot windy summers and short mild winters with little rain. The mean monthly minimum and maximum temperature vary from $12.4 \pm 0.9^{\circ} \mathrm{C}$ in January to $28 \pm 1.2$ in July and $17.5 \pm 0.6^{\circ} \mathrm{C}$ in January to $45 \pm 1.9{ }^{\circ} \mathrm{C}$ in July respectively. The Rainfall is irregular; occurring primarily in winter, and usually does not exceed $3 \mathrm{~mm}$ per year. The average monthly rainfall ranges from a minimum $2.1 \pm 2.4 \mathrm{~mm}$ in November to $3 \mathrm{~mm}$ in January and the relative humidity from 34\% in April to $76.9 \%$ in August. Weather is divided into four well-marked seasons. Summer (May to July), autumn (August to October), and winter (November to January) and spring season (February to April). Native vegetation is characterized by open patches of grasses and forbs. Respectively, camels, sheep and goats are the major agricultural enterprise of farmers of the study area. Climatic data pertaining to maximum, minimum temperature and rainfall was obtained from meteorological station at Shalatin city.

\section{Epidemiological survey:}

The selected animals were visited three times a month in order to collect all relevant information including age, gender of host, number of infested animals and average number of ticks per animal. A total of 882 native breed animals comprised of (551 camels, 225 sheep and 106 goats) were subjected to careful examinations for ticks and tick borne heamoparasites during the period from July. 2009 to Jun. 2010. 


\section{Sampling:}

Tick collection: ticks were collected by detachment of different types of ticks from different parts of the body (ear, eye, neck, belly, anus, scrotum, brisket, udder, vulva, testes, shoulder, tail, and fat tail). All visible ticks were carefully detached by holding it with a curved forceps and turning it anti-clockwise to loose the teeth of the hypostome from the surrounding tissues. Tick infestation was categorized as none, few (1-20 tick per animals), moderate (21-50 tick per animals) or heavy (more than 50 tick per animal). El-Ghali and Hassan (2009).

Tick preservation: The ticks were collected in plastic or glass vials of $25 \mathrm{~mm}$ diameter and $75 \mathrm{~mm}$ long. Engorged and partially engorged females were individually placed in vials which were covered with linen cloth secured in place by rubber bands. Engorged larvae and nymphs were collected in groups of 20 and 10 each, respectively, per vial. Other tick specimens were collected in vials containing 70\% alcohol. These vials were closed with thick plastic stoppers. Fahmy (1980). Permanent mounts of the collected ticks was carried out according to Kruse and Pritchard (1982) while morphological examination and identification were done after key of (Hoogstraal, 1956); (Hoogstraal and Kaiser, 1959); (Karrar et al., 1963); (Walker et al., 2003).

\section{Haemolymph smears, egg smears and salivary glands examination:}

The tick Haemolymph smears: Were obtained from a wound produced by amputating the distal portion of one or more legs. The hemolymph was collected on glass slides which were marked by a grease pencil. Each glass slide was divided into 2 rows. Each row had 5 squares. Ten haemolymph smears were made on each slide, air dried, fixed in methanol stained with Giemsa and examined microscopically (Burgdorfer, 1970). Egg smears. Female ticks from which haemolymph proved to be positive were selected for egg laying. Each female was put in a separate glass tube, incubated and left until egg laying.Egg smears from 5 eggs were prepared daily from the first day till the end of the oviposition period by crushing the egg with a wooden tooth -pick. The smears were air-dried, fixed and stained with diluted Giemsa stain solution and examined with oil -immersion lens (El- Seify, 1980). Dissections of the salivary glands of engorged nymph and moderately fed and unfed adult tick were made for the demonstration and isolation of sporozoites. The parts of the glands were touched lightly onto a 
microscope slide, fixed in methanol and Giemsa-stained (8\%) for the identification of parasitic stage. (Schien and Friedhoff, 1978).

\section{Blood samples:}

Tick infested animals (camels, sheep and goats) were bled from the jugular vein into vacationer tubes containing EDTA as anticoagulant. The tubes were kept on ice during transport to the laboratory, and processed within $3 \mathrm{~h}$ of collection. A thin and thick blood smears were prepared from each blood sample, air-dried, fixed in methanol for 2-3 min, stained with 5\% Giemsa stain and rinsed in two changes of buffered distilled water ( $\mathrm{pH}$ 7.2). The smears were examined by oil immersion lens according to (Lawrence and Thoma, 1987). The recorded tick-borne haemoparasites were identified to genus and where possible species level, according to (Uilenberg, 1981; Levine, 1985; Norval et al., 1992). Quantitative evaluation of parasitaemia (percentage of infected RBCs) was assessed by counting of the number of parasitized erythrocytes present per 1000 cells at a magnification of $\times 1000$ then divided by ten and expressed as parasitaemia percentage (Lawrence and Thoma, 1987).

\section{RESULTS}

\section{Prevalence of tick infestation}

The results of this study over four seasons in Shalatin city are presented in Tables 1-6. Out of 551 camels 154(27.94\%) were infested with a total of 2563 adult ticks, of 225 sheep 41(18.22\%) were infested with 144 adult ticks and of 106 goats 25(23.58\%) were infested with 104 adult ticks. The taxonomic identification of tick specimen collected from the study area revealed the presence of more than one type of tick species on the domestic ruminants in the study area. The species identified in camels were presented in (Tables 5, Fig. 2-5). Hyalomma dromedarii was found to be the predominant tick species $(90.90 \%)$. Other tick species found in low numbers were Hyalomma impeltatum (3.90\%); Hyalomma anatolicum anatolicum (2.93\%) and Amblyomma lepidum (2.26\%) with an average density of 22.91-26.56 ticks per animal. Ticks infestation was more prevalent in male while female animals harbored more ticks infestation than males. Four tick species were found on sheep and goats during the study period. These were Rhipicephalus sp., Haemophysalis sp., H. dromedarii and Hyalomma a. anatolicum. Of these ticks Rhipicephalus 
$s p$., was the most abundant species and constituted (45.14\%) and (44.23\%), of the ticks collected in sheep and goats respectively. The second most abundant species was Haemophysalis sp., and constituted (38.19\% and 38.46\%) in sheep and goats respectively with an average density of (3.82 \& 3.06) and (4.60 \& 3.63) tick/host. Where $H$. dromedarii on sheep and Hyalomma a. anatolicum on goats represented a very small percentage $(16.66 \& 17.30)$ of the collected ticks respectively.

\section{Month wise - prevalence of ticks Camels}

The infested camels were observed throughout the year and increased in number from May to August with peak in August and lowest in January (Table 1).

\section{Sheep and goats}

The infested sheep and goats were observed from May to August with peak in August and lowest in November, no animals were found infested with tick from December- February (Tables 2 and 3), the average number of ticks was also found to be directly proportional to the prevalence percentage i.e. when the prevalence was higher, the intensity of infestation was higher and vice versa.

\section{Age and gender wise- prevalence of ticks}

Higher prevalence was found in females than males as shown in (Tables 1, $2 \& 3$ ), although higher numbers of ticks per animal were found in older animals as compared with younger ones (Table 4).

\section{Sites of tick infestation on studied animals}

Various body sites of the study animals were examined in order to categorize the sites with high affinity for tick infestation. It was found that the udder, scrotum, belly and brisket were the preferred sites for feeding in camels followed by testis, neck, vulva, anus, shoulder, ear and eye. (Table 5 and Fig. 1). In sheep and goats the result showed that hard ticks infestation was highest on fat tail of sheep and tail of goats followed by udder, flank, eye and ear (Table 6 and Fig.1). 


\section{Tick borne haemoparasites}

Haemoparasites in camels: Tick-borne haemoparasites of two genera were detected in camels; Theileria camelensis, and Anaplasma marginale based on morphological characteristics and epidemiological considerations, with an infection rate $48.58 \%$, and $6.49 \%$ (Table, 7, Fig. 6-11).

Haemoparasites in sheep: Four genera of tick-borne haemoparasites were found in blood smears from sheep; Theileria ovis. Babesia sp., Anaplasma marginale, and Eperythrozoon sp., with an infection rate $51.21 \%, 12.20 \%, 14.63 \%$ and $9.76 \%$ respectively. Based on morphological characteristics and epidemiological considerations (Table, 8, Fig. 6-11).

Haemoparasites in goats: Two genera of tick-borne haemoparasites were found in blood smears from goats, Theileria ovis and Anaplasma marginale on morphological characteristics and epidemiological considerations with an infection rate 52\% and 24\% (Table, 9, Fig. 6-11). Parasitaemia of the recoded haemoparasites ranged (2\%-15\%).

Morphological status of the developmental stages in the haemolymph, egg smears and salivary gland of ticks:

Examination of the haemolymph, (Fig. 17-21, 25) and egg smears, (Fig. 22-24) of Hyalomma dromedarii ticks revealed presence of five developmental stages of Theileria (Club, banana, spindle, crescentic and rod forms). The banana forms were the highest, followed by the spindle, club, crescentic and rod forms, while examination of salivary glands of engorged nymph, moderate fed and unfed adult ticks of genus Hyalomma dromedarii revealed the developmental stages of Theileria which penetrating kinetes till spindle-shaped sporozoites (infective stages), (Fig. 12-16). 
Table 1: Month wise-prevalence of ticks' infestation in camels.

\begin{tabular}{|c|c|c|c|c|c|c|c|c|c|c|c|}
\hline \multirow{2}{*}{\multicolumn{2}{|c|}{ Months }} & \multirow[b]{2}{*}{$\begin{array}{c}\text { No. of } \\
\text { exam. } \\
\text { camels }\end{array}$} & \multirow[b]{2}{*}{$\begin{array}{l}\text { No. of } \\
\text { infested } \\
\text { camels }\end{array}$} & \multicolumn{3}{|c|}{ Male animals } & \multicolumn{3}{|c|}{ Female animals } & \multicolumn{2}{|c|}{ Total } \\
\hline & & & & $\begin{array}{c}\text { No.of } \\
\text { animals }\end{array}$ & $\begin{array}{l}\text { No. of } \\
\text { collected } \\
\text { ticks }\end{array}$ & $\begin{array}{c}\text { Mean } \\
/ \\
\text { anima } \\
1\end{array}$ & $\begin{array}{l}\text { No.of } \\
\text { animals }\end{array}$ & $\begin{array}{l}\text { No. of } \\
\text { collected } \\
\text { ticks }\end{array}$ & $\begin{array}{c}\text { Mean } \\
/ \\
\text { anima } \\
1\end{array}$ & $\begin{array}{l}\text { No. of } \\
\text { ticks }\end{array}$ & $\begin{array}{c}\text { Mean } \\
/ \\
\text { anima } \\
1\end{array}$ \\
\hline Jul & \multirow{6}{*}{$\begin{array}{l}2 \\
0\end{array}$} & 60 & 24 & 16 & 325 & 20.31 & 8 & 225 & 28.13 & 550 & 22.92 \\
\hline Aug & & 56 & 23 & 14 & 421 & 30.07 & 9 & 190 & 21.11 & 611 & 26.57 \\
\hline Sept & & 40 & 14 & 10 & 120 & 12 & 4 & 82 & 20.5 & 202 & 14.43 \\
\hline Oct & & 42 & 9 & 5 & 82 & 16.4 & 4 & 75 & 18.75 & 157 & 17.44 \\
\hline Nov & & 45 & 7 & 3 & 43 & 14.33 & 4 & 28 & 7 & 71 & 10.14 \\
\hline Dec & & 43 & 4 & 3 & 14 & 4.67 & 1 & 8 & 8 & 22 & 5.50 \\
\hline Jan & \multirow{6}{*}{$\begin{array}{l}2 \\
0 \\
1 \\
0\end{array}$} & 39 & 5 & 3 & 14 & 4.67 & 2 & 9 & 4.5 & 23 & 4.60 \\
\hline Feb & & 40 & 5 & 2 & 13 & 6.5 & 3 & 28 & 9.33 & 41 & 8.20 \\
\hline Mar & & 41 & 10 & 4 & 40 & 10 & 6 & 72 & 12 & 112 & 11.20 \\
\hline Apr & & 50 & 13 & 8 & 89 & 11.13 & 5 & 68 & 13.6 & 157 & 12.08 \\
\hline May & & 45 & 18 & 11 & 105 & 9.55 & 7 & 140 & 20 & 245 & 13.61 \\
\hline Jun & & 50 & 22 & 14 & 184 & 13.14 & 8 & 188 & 23.5 & 372 & 16.90 \\
\hline Total & & 551 & $\begin{array}{c}154 \\
(27.94)\end{array}$ & 93 & 1450 & 15.59 & 61 & 1113 & 18.25 & 2563 & 16.64 \\
\hline
\end{tabular}


Table 2: Month wise-prevalence of ticks' infestation in sheep

\begin{tabular}{|c|c|c|c|c|c|c|c|c|c|c|c|}
\hline \multirow{2}{*}{\multicolumn{2}{|c|}{ Months }} & \multirow{3}{*}{$\begin{array}{c}\begin{array}{c}\text { No. of } \\
\text { exam. } \\
\text { sheep }\end{array} \\
22 \\
\end{array}$} & \multirow{3}{*}{$\begin{array}{c}\begin{array}{c}\text { No. of } \\
\text { infested } \\
\text { sheep }\end{array} \\
9 \\
\end{array}$} & \multicolumn{3}{|c|}{ Male animals } & \multicolumn{3}{|c|}{ Female animals } & \multicolumn{2}{|c|}{ Total } \\
\hline & & & & No.of & No. of & Mean & No.of & No. of & Mean & No. of & Mean \\
\hline Jul & \multirow{6}{*}{$\begin{array}{l}2 \\
0 \\
0 \\
9\end{array}$} & & & 6 & 10 & 1.67 & 3 & 21 & 7 & 31 & 3.44 \\
\hline $\begin{array}{l}\text { Augu } \\
\text { st }\end{array}$ & & 20 & 9 & 4 & 19 & 4.75 & 5 & 21 & 4.2 & 40 & 4.44 \\
\hline Sept & & 16 & 5 & 4 & 10 & 2.5 & 1 & 7 & 7 & 17 & 3.4 \\
\hline Oct & & 14 & 4 & 2 & 5 & 2.5 & 2 & 3 & 2 & 9 & 2.25 \\
\hline Nov & & 13 & 2 & 1 & 2 & 2 & 1 & 4 & 3 & 5 & 2.5 \\
\hline Dec & & 17 & 0 & 0 & 0 & 0 & 0 & 0 & 0 & 0 & 0 \\
\hline Jan & \multirow{6}{*}{$\begin{array}{l}2 \\
0 \\
1 \\
0\end{array}$} & 19 & 0 & 0 & 0 & 0 & 0 & 0 & 0 & 0 & 0 \\
\hline Feb & & 12 & 0 & 0 & 0 & 0 & 0 & 0 & 0 & 0 & 0 \\
\hline Mar & & 20 & 2 & 1 & 3 & 3 & 1 & 4 & 4 & 7 & 3.5 \\
\hline Apr & & 17 & 2 & 1 & 3 & 3 & 1 & 3 & 3 & 6 & 3 \\
\hline May & & 22 & 3 & 2 & 6 & 3 & 1 & 4 & 4 & 10 & 3.33 \\
\hline Jun & & 23 & 5 & 3 & 10 & 3.33 & 2 & 9 & 4.5 & 19 & 3.8 \\
\hline Total & & 225 & $\begin{array}{c}41 \\
(18.22) \\
\end{array}$ & 24 & 68 & 2.83 & 17 & 76 & 4.47 & 144 & 3.51 \\
\hline
\end{tabular}


Table 3: Month wise-prevalence of ticks' infestation in goats.

\begin{tabular}{|c|c|c|c|c|c|c|c|c|c|c|c|}
\hline \multirow{2}{*}{\multicolumn{2}{|c|}{ Months }} & \multirow{3}{*}{$\begin{array}{c}\begin{array}{c}\text { No. of } \\
\text { exam. } \\
\text { goats }\end{array} \\
14 \\
\end{array}$} & \multirow{3}{*}{$\begin{array}{c}\begin{array}{c}\text { No. of } \\
\text { infested } \\
\text { goats }\end{array} \\
5\end{array}$} & \multicolumn{3}{|c|}{ Male animals } & \multicolumn{3}{|c|}{ Female animals } & \multicolumn{2}{|c|}{ Total } \\
\hline & & & & \multirow{2}{*}{\begin{tabular}{|c|}
$\begin{array}{c}\text { No.of } \\
\text { animals }\end{array}$ \\
4 \\
\end{tabular}} & \multirow{2}{*}{ 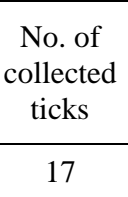 } & \multirow{2}{*}{$\begin{array}{c}\text { Mean } \\
\text { / } \\
\text { animal } \\
4.25\end{array}$} & \multirow{2}{*}{\begin{tabular}{|c|}
$\begin{array}{l}\text { No.of } \\
\text { animals }\end{array}$ \\
1 \\
\end{tabular}} & \multirow{2}{*}{$\begin{array}{c}\begin{array}{c}\text { No. of } \\
\text { collected } \\
\text { ticks }\end{array} \\
5 \\
\end{array}$} & \multirow{2}{*}{$\begin{array}{c}\text { Mean } \\
/ \\
\text { animal } \\
5\end{array}$} & \multirow{2}{*}{$\begin{array}{c}\begin{array}{c}\text { No. of } \\
\text { ticks }\end{array} \\
22 \\
\end{array}$} & \multirow{2}{*}{$\begin{array}{c}\begin{array}{c}\text { Mean } \\
/ \\
\text { animal }\end{array} \\
4.4\end{array}$} \\
\hline Jul & & & & & & & & & & & \\
\hline $\begin{array}{l}\text { Aug } \\
\text { ust }\end{array}$ & \multirow{5}{*}{$\begin{array}{l}20 \\
09\end{array}$} & 11 & 4 & 3 & 16 & 5.33 & 1 & 5 & 5 & 21 & 5.25 \\
\hline Sept & & 9 & 2 & 1 & 3 & 3 & 1 & 3 & 3 & 6 & 3 \\
\hline Oct & & 8 & 2 & 1 & 2 & 2 & 1 & 3 & 3 & 5 & 2.5 \\
\hline Nov & & 6 & 1 & 0 & 0 & 0 & 1 & 2 & 2 & 2 & 2 \\
\hline Dec & & 5 & 0 & 0 & 0 & 0 & 0 & 0 & 0 & 0 & 0 \\
\hline Jan & \multirow{6}{*}{$\begin{array}{l}20 \\
10\end{array}$} & 9 & 0 & 0 & 0 & 0 & 0 & 0 & 0 & 0 & 0 \\
\hline Feb & & 8 & 0 & 0 & 0 & 0 & 0 & 0 & 0 & 0 & 0 \\
\hline Mar & & 7 & 1 & 1 & 3 & 3 & 0 & 0 & 0 & 3 & 3 \\
\hline Apr & & 7 & 2 & 1 & 3 & 3 & 1 & 5 & 5 & 8 & 4 \\
\hline May & & 9 & 2 & 1 & 3 & 3 & 1 & 3 & 3 & 6 & 3 \\
\hline Jun & & 13 & 6 & 4 & 20 & 5 & 2 & 11 & 5.5 & 31 & 5.17 \\
\hline Total & & 106 & $\begin{array}{c}25 \\
(23.58 \%)\end{array}$ & 16 & 67 & 4.18 & 9 & 37 & 4.11 & 104 & 4.16 \\
\hline
\end{tabular}


Table 4: Age wise-prevalence of tick infestation in studied animals.

\begin{tabular}{|c|c|c|c|c|}
\hline Animal category & Age by year & $\begin{array}{c}\text { No. of exam. } \\
\text { animals }\end{array}$ & $\begin{array}{c}\text { Mean of animal } \\
\text { infested }\end{array}$ & Prevalence \\
\hline \multirow{3}{*}{ Camels } & $1-4$ & 136 & 28 & 20.59 \\
\cline { 2 - 5 } & $5-8$ & 188 & 52 & 27.66 \\
\cline { 2 - 5 } & $9-12$ & 227 & 74 & 32.60 \\
\hline \multirow{3}{*}{ Sheep } & $<1$ & 45 & 4 & 8.89 \\
\cline { 2 - 5 } & $1-3$ & 88 & 15 & 17.05 \\
\hline \multirow{3}{*}{ Goats } & $4-5$ & 92 & 22 & 23.91 \\
\cline { 2 - 5 } & $1-3$ & 22 & 1 & 4.55 \\
\cline { 2 - 5 } & $4-5$ & 47 & 9 & 19.15 \\
\hline
\end{tabular}


Table 5: Sites of tick infestation in camels.

\begin{tabular}{|c|c|c|c|c|c|c|c|c|c|c|c|c|c|c|}
\hline \multirow{2}{*}{$\begin{array}{l}\text { Animal } \\
\text { Species }\end{array}$} & \multirow{2}{*}{$\begin{array}{c}\text { No. of } \\
\text { infested } \\
\text { animal }\end{array}$} & \multirow{2}{*}{ Tick species } & \multicolumn{12}{|c|}{ Body sites } \\
\hline & & & $\mathrm{E}$ & ey & $\mathrm{n}$ & bri & $\mathrm{b}$ & $\mathrm{u}$ & $\mathrm{s}$ & A & $\mathrm{v}$ & te & Sh. & Samples \\
\hline \multirow{13}{*}{ Camel } & \multirow[t]{3}{*}{$\begin{array}{c}125 \\
(81.16 \%)\end{array}$} & $\begin{array}{l}\text { H. dromedarii } \\
(\hat{0})\end{array}$ & 92 & 78 & 195 & 209 & 210 & 220 & 217 & 171 & 190 & 203 & 145 & $\begin{array}{c}1930 \\
(75.30 \% \\
\end{array}$ \\
\hline & & $\begin{array}{l}\text { H. dromedarii } \\
(+)\end{array}$ & 41 & 35 & 18 & 39 & 43 & 39 & 30 & 50 & 39 & 45 & 21 & $\begin{array}{c}400 \\
(15.61 \% \\
\end{array}$ \\
\hline & & $\begin{array}{l}\text { H. dromedarii } \\
(\hat{0}+q)\end{array}$ & 133 & 113 & 213 & 248 & 253 & 259 & 247 & 221 & 229 & 248 & 166 & $\begin{array}{c}2330 \\
(90.90 \% \\
\end{array}$ \\
\hline & \multirow[t]{3}{*}{$\begin{array}{c}15 \\
(9.74 \%)\end{array}$} & $\begin{array}{l}\text { H. impeltatum } \\
(\lesssim)\end{array}$ & 4 & 2 & 10 & 8 & 5 & 5 & 6 & 4 & 8 & 7 & 4 & $\begin{array}{c}63 \\
(2.46 \%) \\
\end{array}$ \\
\hline & & $\begin{array}{l}\text { H. impeltatum } \\
\text { () }\end{array}$ & 2 & 4 & 1 & 9 & 4 & 1 & 1 & 2 & 4 & 5 & 4 & $\begin{array}{c}37 \\
(1.44 \%) \\
\end{array}$ \\
\hline & & $\begin{array}{l}\text { H. impeltatum } \\
(\hat{\jmath}+\stackrel{+}{0})\end{array}$ & 6 & 6 & 11 & 17 & 9 & 6 & 7 & 6 & 12 & 12 & 8 & $\begin{array}{c}100 \\
(3.90 \%) \\
\end{array}$ \\
\hline & \multirow[t]{3}{*}{$\begin{array}{c}8 \\
(5.19 \%)\end{array}$} & $\begin{array}{l}\text { H. a anatolicum } \\
(\text { đ) }\end{array}$ & 3 & 2 & 6 & 4 & 4 & 6 & 4 & 4 & 7 & 5 & 5 & $\begin{array}{c}50 \\
(1.95 \%) \\
\end{array}$ \\
\hline & & $\begin{array}{l}\text { H. a anatolicum } \\
\text { (ㅇ) }\end{array}$ & 3 & 1 & 2 & 2 & 2 & 2 & 4 & 2 & 3 & 2 & 2 & $\begin{array}{c}25 \\
(0.96 \%) \\
\end{array}$ \\
\hline & & $\begin{array}{l}\text { H. a anatolicum } \\
(\hat{\partial}+9)\end{array}$ & 6 & 3 & 8 & 6 & 6 & 8 & 8 & 6 & 10 & 7 & 7 & $\begin{array}{c}75 \\
(2.93 \%) \\
\end{array}$ \\
\hline & & $\begin{array}{l}\text { Hyalomma } \\
\text { nymphs }\end{array}$ & 3 & 10 & 200 & 100 & 150 & 50 & 170 & 31 & 30 & 65 & 79 & $\begin{array}{c}888 \\
(34.64 \% \\
\end{array}$ \\
\hline & \multirow[t]{3}{*}{$\begin{array}{c}6 \\
(3.89 \%)\end{array}$} & $\begin{array}{l}\text { Amblyomma } \\
\text { lepidum }(\precsim)\end{array}$ & 3 & 2 & 3 & 4 & 5 & 3 & 4 & 3 & 3 & 4 & 3 & $\begin{array}{c}37 \\
(1.44 \%) \\
\end{array}$ \\
\hline & & $\begin{array}{l}\text { Amblyomma } \\
\text { lepidum }(+\rightarrow)\end{array}$ & 1 & 1 & 2 & 1 & 2 & 3 & 3 & 2 & 2 & 2 & 2 & $\begin{array}{c}21 \\
(0.82 \%) \\
\end{array}$ \\
\hline & & $\begin{array}{l}\text { Amblyomma } \\
\text { lepidum }(\hat{\jmath}+q)\end{array}$ & 4 & 3 & 5 & 5 & 7 & 6 & 7 & 5 & 5 & 6 & 5 & $\begin{array}{c}58 \\
(2.26 \%)\end{array}$ \\
\hline
\end{tabular}


$\mathrm{e}=$ ear, ey = eye, $\mathrm{n}=$ neck, $\mathrm{b}=$ belly, $\mathrm{a}=$ anus, $\mathrm{s}=$ scrotum, bri $=$ brisket, $\mathrm{u}=$ udder, $\mathrm{v}=$ vulva, te $=$ testes, sh= shoulder

Table 6: Sites of tick infestation in sheep and goats.

\begin{tabular}{|c|c|c|c|c|c|c|c|c|c|c|c|c|c|c|}
\hline \multirow{2}{*}{$\begin{array}{l}\text { Animal } \\
\text { Species }\end{array}$} & \multirow{2}{*}{$\begin{array}{l}\text { No. of } \\
\text { infested } \\
\text { animal }\end{array}$} & \multirow{2}{*}{ Tick species } & \multicolumn{12}{|c|}{ Body sites } \\
\hline & & & e & ey & f & $\mathrm{ab}$ & $\mathrm{a}$ & $\mathrm{ft}$ & $\mathrm{t}$ & $\mathrm{U}$ & $\mathbf{v}$ & te & $\mathbf{S}$ & Samples \\
\hline \multirow{5}{*}{ sheep } & 17 & $\begin{array}{l}\text { Rhipicephalus } \\
\text { spp. }\end{array}$ & 5 & 5 & 10 & 6 & 4 & 15 & 0 & 10 & 4 & 0 & 6 & $\begin{array}{c}65 \\
(45.14 \%\end{array}$ \\
\hline & 18 & $\begin{array}{l}\text { Haemophysalis } \\
\text { spp. }\end{array}$ & 3 & 4 & 8 & 4 & 3 & 16 & 0 & 9 & 3 & 0 & 5 & $\begin{array}{c}55 \\
(38.19 \%\end{array}$ \\
\hline & \multirow{3}{*}{6} & $\begin{array}{l}\text { H. dromedarii } \\
(0)\end{array}$ & 1 & 1 & 3 & 1 & 2 & 6 & 0 & 1 & 0 & 0 & 1 & 16 \\
\hline & & $\begin{array}{l}\text { H. dromedarii } \\
(\text { ( ) }\end{array}$ & 1 & 1 & 1 & 0 & 0 & 4 & 0 & 1 & 0 & 0 & 0 & 8 \\
\hline & & $\begin{array}{l}\text { H. dromedarii } \\
(\hat{\delta}+q)\end{array}$ & 2 & 2 & 4 & 1 & 2 & 10 & 0 & 2 & 0 & 0 & 1 & $\begin{array}{c}24 \\
(16.67 \%\end{array}$ \\
\hline \multirow{5}{*}{ Goat } & 10 & $\begin{array}{l}\text { Rhipicephalus } \\
\text { spp. }\end{array}$ & 4 & 4 & 8 & 0 & 1 & 0 & 13 & 8 & 6 & 2 & 0 & $\begin{array}{c}46 \\
(44.23 \%\end{array}$ \\
\hline & 11 & $\begin{array}{l}\text { Haemophysalis } \\
\text { spp. }\end{array}$ & 4 & 3 & 6 & 0 & 3 & 0 & 10 & 7 & 5 & 2 & 0 & $\begin{array}{c}40 \\
(38.46 \%\end{array}$ \\
\hline & \multirow{3}{*}{4} & H. a anatolicum & 1 & 1 & 2 & 0 & 2 & 0 & 2 & 2 & 1 & 0 & 0 & 11 \\
\hline & & $\begin{array}{l}\text { H. a anatolicum } \\
(9)\end{array}$ & 1 & 1 & 0 & 0 & 1 & 0 & 2 & 1 & 1 & 0 & 0 & 7 \\
\hline & & $\begin{array}{l}\text { H. a anatolicum } \\
(\delta+q)\end{array}$ & 2 & 2 & 2 & 0 & 3 & 0 & 4 & 3 & 2 & 0 & 0 & $\begin{array}{c}18 \\
(17.30 \%\end{array}$ \\
\hline
\end{tabular}


$\mathrm{e}=$ ear, $\mathrm{ey}=$ eye, $\mathrm{n}=$ neck, $\mathrm{ab}=$ abdomen, $\mathrm{a}=$ anus, $\mathrm{f}=$ flank, $\mathrm{p}=$ prepuce $\mathrm{u}=$ udder, $\mathrm{v}=$ vulva, $\mathrm{te}=$ testes, $\mathrm{s}=$ shoulder, $\mathrm{t}=$ tail, $\mathrm{ft}=$ fat tail.

Table 7: Tick borne parasites identified in camels by month among 154 tick infested camels).

\begin{tabular}{|c|c|c|c|c|c|c|c|c|c|c|c|c|c|c|}
\hline $\begin{array}{r}\text { Date of } \\
\text { sampling }\end{array}$ & Jul & August & Sep & Oct & Nov & Dec & Jan & Feb & Mar & Apr & May & Jun & No. & $\%$ \\
\hline $\begin{array}{l}\text { Theileria } \\
\text { camelensis }\end{array}$ & 15 & 18 & 5 & 3 & 2 & 0 & 0 & 1 & 3 & 4 & 10 & 13 & 74 & \multirow{3}{*}{48.58} \\
\hline$\%$ samples +ve & & & & & & & & & & & & & & \\
\hline $\begin{array}{l}\text { Mean level } \\
\text { parasitaemia }\end{array}$ & +++ & +++ & + & + & + & 0 & 0 & 0 & 0 & ++ & ++ & +++ & & \\
\hline $\begin{array}{l}\text { Anaplasma } \\
\text { marginale }\end{array}$ & 2 & 4 & 1 & 0 & 0 & 0 & 0 & 0 & 0 & 0 & 1 & 2 & 10 & \multirow{3}{*}{6.49} \\
\hline$\%$ samples + ve & & & & & & & & & & & & & & \\
\hline $\begin{array}{l}\text { Mean level } \\
\text { parasitaemia }\end{array}$ & ++ & + & + & 0 & 0 & 0 & 0 & 0 & 0 & 0 & 0 & +++ & & \\
\hline
\end{tabular}


Table 8: Tick borne parasites identified in sheep by month (among 41tick infested sheep).

\begin{tabular}{|c|c|c|c|c|c|c|c|c|c|c|c|c|c|c|}
\hline Parasite spp. & Jul & August & Sep & Oct & Nov & Dec & Jan & Feb & Mar & Apr & May & Jun & No. & $\%$ \\
\hline Theileria ovis & 6 & 7 & 1 & 1 & 0 & 0 & 0 & 0 & 0 & 1 & 2 & 3 & 21 & \multirow{3}{*}{51.21} \\
\hline \multicolumn{14}{|l|}{$\%$ samples +ve } & \\
\hline $\begin{array}{l}\text { Mean level } \\
\text { parasitaemia }\end{array}$ & ++++ & ++++ & ++ & ++ & 0 & 0 & 0 & 0 & 0 & + & ++ & +++ & & \\
\hline Babesia sp., & 1 & 1 & 1 & 0 & 0 & 0 & 0 & 0 & 0 & 0 & 1 & 1 & 5 & \multirow{3}{*}{12.20} \\
\hline \multicolumn{14}{|l|}{$\%$ samples +ve } & \\
\hline $\begin{array}{l}\text { Mean level } \\
\text { parasitaemia }\end{array}$ & + & + & 0 & 0 & 0 & 0 & 0 & 0 & 0 & 0 & ++ & ++ & & \\
\hline $\begin{array}{l}\text { Anaplasma } \\
\text { marginale }\end{array}$ & 1 & 1 & 1 & 1 & 0 & 0 & 0 & 0 & 0 & 0 & 1 & 1 & 6 & \multirow{3}{*}{14.63} \\
\hline$\%$ samples +ve & + & + & 0 & 0 & 0 & 0 & 0 & 0 & 0 & 0 & ++ & ++ & & \\
\hline $\begin{array}{l}\text { Mean level } \\
\text { parasitaemia }\end{array}$ & ++ & ++ & + & ++ & 0 & 0 & 0 & 0 & 0 & + & ++ & +++ & & \\
\hline $\begin{array}{l}\text { Eperythrozoon } \\
\text { sp. }\end{array}$ & 1 & 1 & 0 & 0 & 0 & 0 & 0 & 0 & 0 & 0 & 1 & 1 & 4 & \multirow{3}{*}{9.76} \\
\hline$\%$ samples +ve & & & & & & & & & & & & & & \\
\hline $\begin{array}{l}\text { Mean level } \\
\text { parasitaemia }\end{array}$ & ++ & +++ & + & + & 0 & 0 & 0 & 0 & 0 & 0 & 0 & 0 & & \\
\hline
\end{tabular}


Table 9: Tick borne parasites identified in Goat by month (among 25 tick infested goats).

\begin{tabular}{|c|c|c|c|c|c|c|c|c|c|c|c|c|c|c|}
\hline $\begin{array}{l}\text { Date of } \\
\text { sampling }\end{array}$ & & & & & & & & & & & & & & \\
\hline Parasite spp. & Jul & August & Sep & Oct & Nov & Dec & Jan & Feb & Mar & Apr & May & Jun & No. & $\%$ \\
\hline Theileria ovis & 2 & 3 & 1 & 0 & 0 & 0 & 0 & 0 & 1 & 1 & 2 & 3 & 13 & \multirow{3}{*}{$52 \%$} \\
\hline$\%$ samples +ve & & & & & & & & & & & & & & \\
\hline $\begin{array}{l}\text { Mean level } \\
\text { parasitaemia }\end{array}$ & ++ & ++++ & + & 0 & 0 & 0 & 0 & 0 & 0 & + & +++ & +++ & & \\
\hline $\begin{array}{l}\text { Anaplasma } \\
\text { marginale }\end{array}$ & 1 & 1 & 1 & 0 & 0 & 0 & 0 & 0 & 0 & 1 & 1 & 1 & 6 & \multirow{3}{*}{$24 \%$} \\
\hline$\%$ samples +ve & & & & & & & & & & & & & & \\
\hline $\begin{array}{l}\text { Mean level } \\
\text { parasitaemia }\end{array}$ & + & + & 0 & 0 & 0 & 0 & 0 & 0 & 0 & + & ++ & +++ & & \\
\hline
\end{tabular}


Assiut Vet. Med. J. Vol. 57 No. 130 July 2011
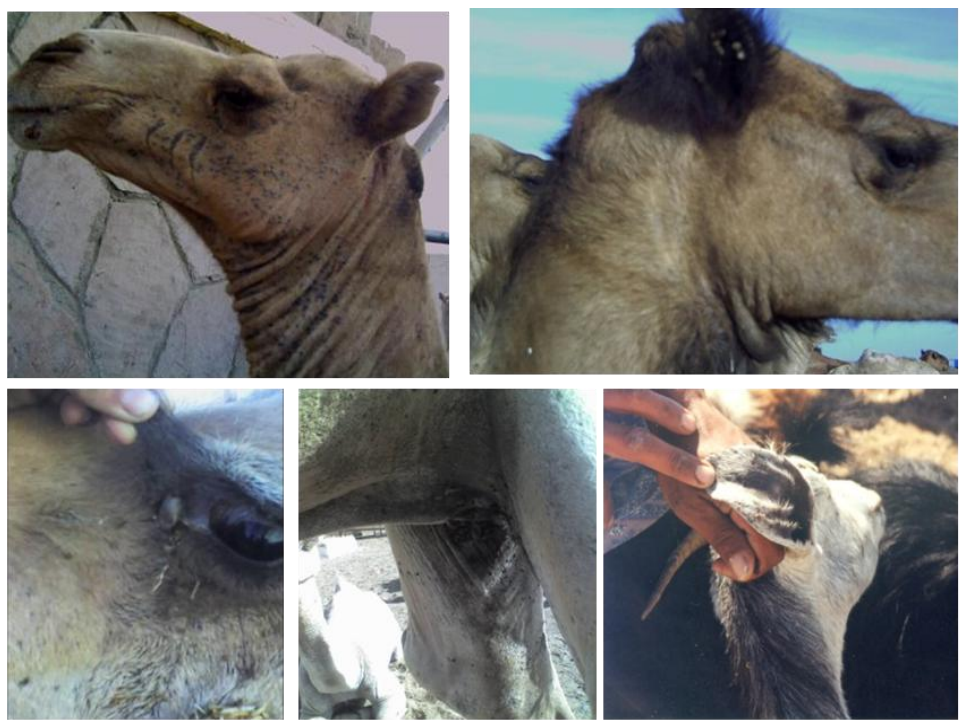

Fig. (1)
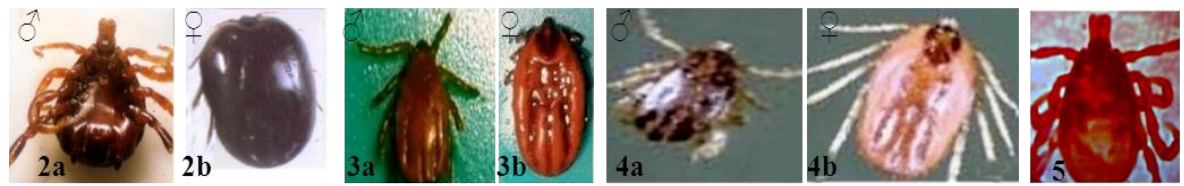

Figs. (2-5)
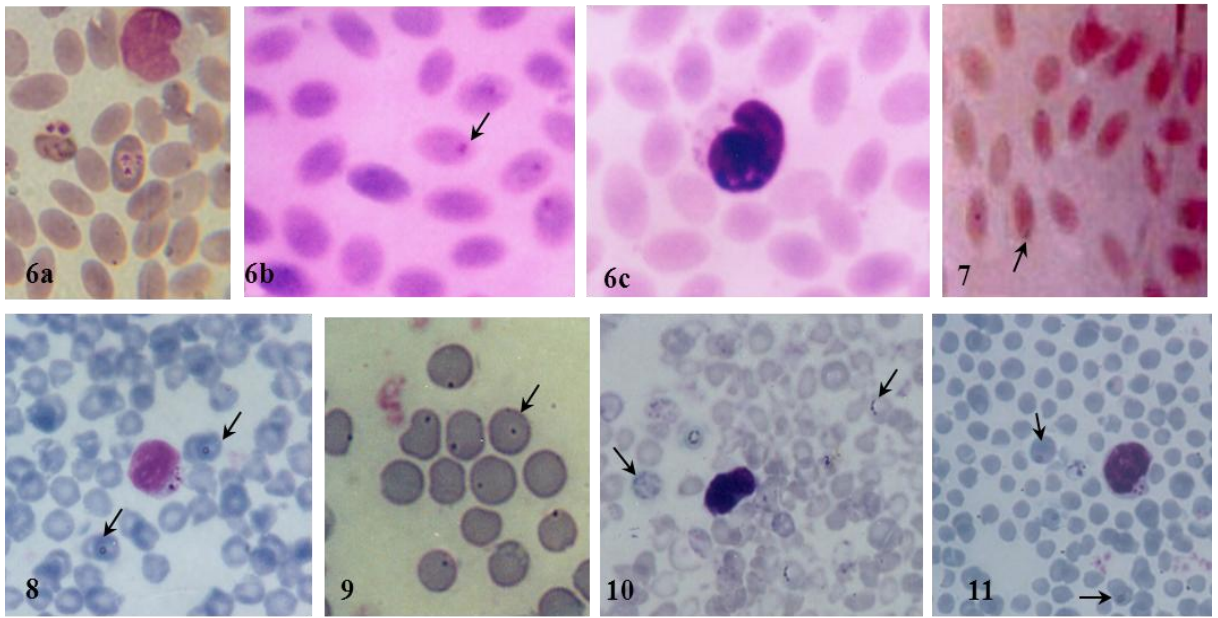

Figs. (6-11) 
Assiut Vet. Med. J. Vol. 57 No. 130 July 2011
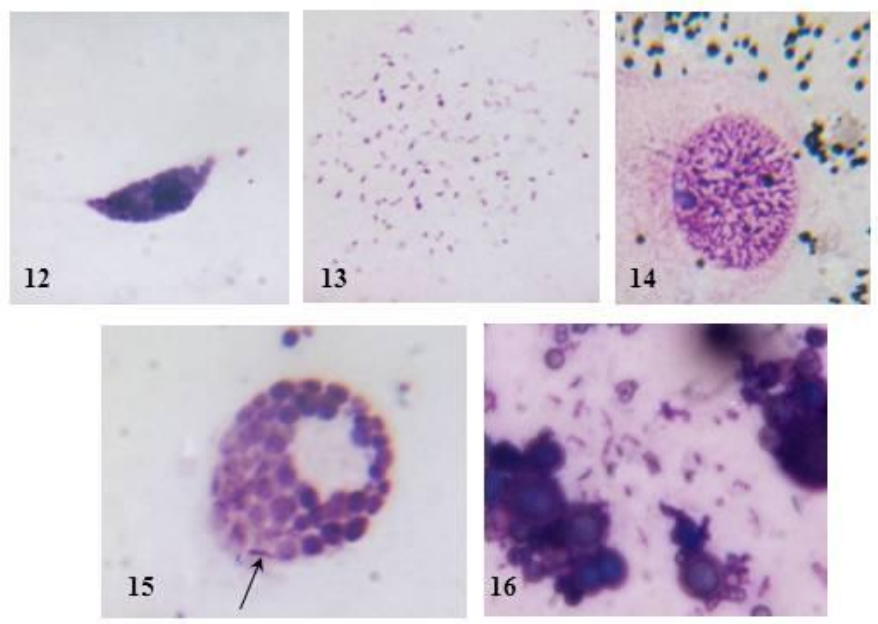

Figs. (12-16)
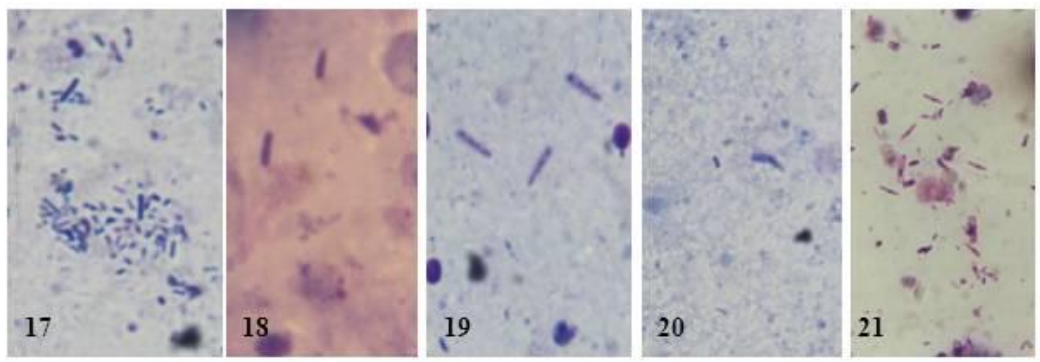

Figs. (17-21)
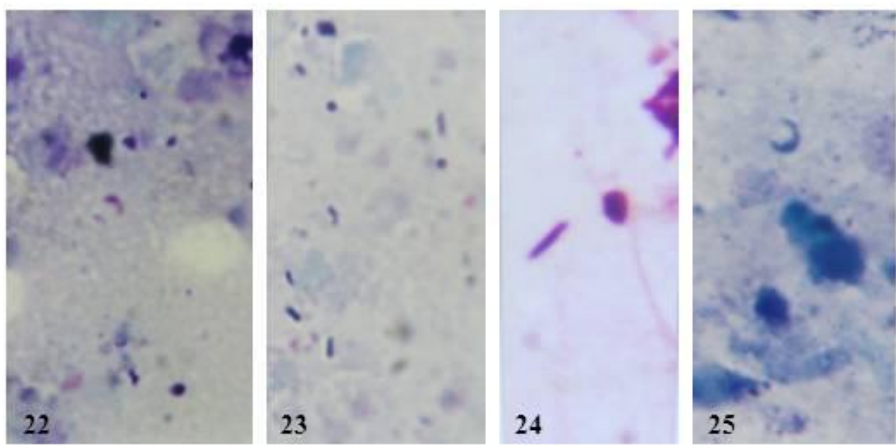

Figs. (22-25) 


\section{LEGEND OF FIGURES}

Fig. 1: Tick infestation sites.

Fig. 2-5: Tick species, 2- Hyalomma dromedarii (ふ, †);

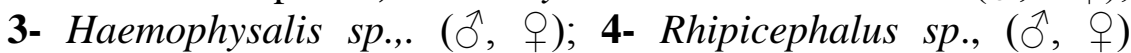
5- Hyalomma anat. Anatolicum ( $\lesssim$ ).

Fig. 6-11: Tick borne haemoparasites, Giemsa stain, x100, 6- Theileria camelensis in blood film of Camelus dromedarii. a) Intracellular trophozoite (low parasitaemia). b) Intracellular trophozoite (high parasitaemia). c) Koch's blue bodies. 7- Anaplasma marginale in Camelus dromedarii blood film $(\rightarrow)$. 8- Theleria ovis in sheep blood film, showing intracellular trophozoite $(\rightarrow)$ \& Koch's blue bodies. 9) - Anaplasma marginale in sheep blood film showing high parasitaemia $(\rightarrow)$ 10- Eperthrozoon sp., in sheep blood film, in the form of minute granules on the surface of RBCs. 11- Theleria ovis in goat blood film, showing intracellular trophozoite $(\rightarrow)$ \& Koch's blue bodies.

Fig. 12-16: Giemsa stained developmental stages Theileria camelensis in salivary gland of feeding Hyalomma dromedarii. Giemsa stain, $\mathrm{x} 100,12-$ Penetrating kinete to salivary gland and give rise to multinucleated sporonts. 13 Diverse nuclear fragments of the sporonts in division. 14 Multinucleated sporont which divided into numerous small sporoblasts which form sporozoites by budding process at their periphery. 15- Polymorphous multinucleated sporoblast with sporozoite at the periphery $(\rightarrow)$. 16- Single spindle shaped sporozoites (infectious stage).

Fig. 17-21: Giemsa stained developmental stages of blood parasites, Theileria camelensis, in haemolymph of feeding Hyalomma dromedarii. Giemsa stain, x100, 17- Multinucleated vermicules. 18- Small rod-shaped vermicule. 19- Large rod -shaped vermicule. 20- Banana-shaped vermicule. 21- Spindle-shaped vermicule.

Fig. 22-25: Giemsa stained developmental stages of Theileria camelensis in eggs of Hyalomma dromedarii ticks,. Giemsa stain, x100, 22- Comma-shaped vermicule (immature stage) in eggs of Hyalomma dromedarii ticks. 23- Small rod-shaped vermicule in eggs of $H$. dromedarii ticks. 24- Large rod-shaped vermicule in eggs of $H$. dromedarii ticks. 25- Cresentric form in haemolymph of $H$. dromedarii ticks. 


\section{DISCUSSION}

This study was conducted in a semi- arid desert zone where rainfall is low and of short duration (Nov-Jan). Results of the present study revealed that examination of 882 of the various domestic animals species, of which 551 were camels, 225 sheep and 106 goats in Shalatin city, Red Sea governorate, Egypt. Out of 551 camels 154 (27.94\%) were found infested with $H$. dromedarii represented the main tick species infesting camels followed by $H$. impeltatum, $H$. a. anatolicum and Amblyomma lepidum, These results were in agreement with, the results obtained by Njanja et al. (1991); Van Straten and Jongejan (1993); Diab et al. (2001); Abdel-Baki (2001); Mazyad and Khalaf (2002); Iqbal (2005); Mamak et al. (2006). It was also found that $H$. dromedarii females were the main tick species found engorged on the camels, while females of the other species; only partially engorged. This could be due to the fact that camels were not the preferred hosts of the latter tick species. These results coincided with the results recorded by Diab et al. (2001) in Egypt and El-Ghalii and Hassan (2009) in Sudan. The number of obtained Amblyomma ticks in camels was lower than that of Hyalomma species in agreement with that mentioned by El-Refaii and Wahba (2003) in camels and Hoogstraal (1956) in cattle. This observation has been also noted by Hadani et al. (1996) in sheep, but in very low number and also referred that Amblyomma may be introduced by migrating birds from Africa.

In the present study, $41(18.22 \%)$ out of 225 sheep and $25(23.58 \%)$ out of 106 goats infested with Rhipcephalus sp., and Haemophysalis sp., followed by $H$. dromedarii in sheep and $H$. $a$. anatolicum in goats. These results coincided with those recorded by Osman (1997); Abdel-Baki (2001); Mohammad and Ali (2006); Mamak et al. (2006); Al-Khalifa et al. (2007); Omer et al. (2007); Sajid et al. (2008); Abunna et al. (2009). In general with the exception of camels, tick burdens on sheep and goats were low. Seasonality of ticks infestation was observed in May, Jun, July and August. Similar results were observed by Diab et al. (2001) they reported that high tick infestations in Egypt occurred during March to November, while Zeleke, and Bekele, (2004) reported that highest tick infestation occur during months of July and August. In the present study, there was no clear pattern of seasonality specially in camels where ticks were found throughout the year but it was observed that the highest infestation occurred from May to August. These results agreed with that reported by 
El-Ghalii and Hassan (2009). This could be attributed to the fact that the non-parasitic flat stages could survive well during winter which was reflected as infestations during the following summer. On the other hand, it was observed that ticks do not go into diapause during winter when ambient temperature drops to about $10{ }^{\circ} \mathrm{C}$. Regarding the seasonal incidence of tick infestation in sheep and goats, it was noticed that highest infestation occurred from May- August., no animals were found infested with ticks from (December- February). Nearly similar results were recorded by Sajid (2007); Mohammad and Ali (2006). The prevalence and general indices of ticks showed difference related to the locality of their host, seasonal changes in the general indices of some ectoparasites paralleled for the peak activity of hard ticks which is from May-August.

Our study revealed that female animals carried more ticks than males. This was true for all adult tick species. Similar findings were recorded by El-Ghali and Hassan (2009). Pregnancy and lactation stress may lower the resistance of females to tick infestation (Ali, 2004). However, this was not true for nymphs as both genders of camels carried loads the differences of which were not significant. This could be due to differences in resistance of male and female camels against larvae that feed and moult on the host, but not against nymphs El-Ghali and Hassan (2009). The current study revealed that male ticks of all species outnumbered females. Similar results were reported by Hoogstraal (1956); Kaiser et al. (1988); El-Ghali and Hassan (2009). This is due to the fact that females detach from the hosts after a few days of feeding to oviposit while males remain for several weeks before dropping (Hoogstraal, 1956). Concerning the sites of tick infestation among studied animals. It was found that the udder, scrotum, belly and brisket were the preferred sites for feeding in camels followed by testis, neck, vulva, anus, shoulder, ear and eye. Similar observation was recorded by El-Ghali and Hassan (2009). However in sheep and goats the predilection sites were fat tail in sheep and tail in goats followed by udder, flank, eye and ear this results agree with the results obtained by Sajid (2007). Predilection site of the infestation may vary with tick species and with animal host. Generally most of ticks have been reported to infest the sites with thinner skin and shorter hair. this predilection is suitable for ticks because it allow easy penetration of their mouth parts into blood vessel for feeding Walker et al. (2003); Sajid (2007). On the other hand, the prevalence of tick infestation has been found to be higher in 
the study area; this may be attributed to the high temperature and warmer seasons, which are suitable for growth of tick population.

Giemsa-stained blood smears prepared monthly from camels, sheep and goats positive for tick infestation were examined for presence of tick-borne haemoparasites. Two genera were observed in camels; Theileria camelensis, (48.58\%) and Anaplasma marginale (6.49\%) and Parasitaemia, represented by parasites -infected erythrocytes, ranged from less than $2 \%$ to $10 \%$. These results were in agreement with those obtained by Nassar (1992); El-Refaii et al. (1998); El Kammah et al. (2001); E1-Fayoumy et al. (2005); Alsaad (2009); Hamed et al. (2011). In sheep four genera of tick-borne haemoparasites were found Theileria ovis., Babesia sp., Anaplasma marginale and Eperythrozoon sp., Based on morphological characteristics and epidemiological considerations. Theileria ovis was the most common parasite (51.21\%). followed by Anaplasma marginle (14.63\%), Babesia sp., (12.20\%) and Eperythrozoon spp. (9.76\%), where in goats two genera of tick-borne haemoparasites were found Theileria ovis (52 \%) and Anaplasma marginale (24\%) on morphological characteristics and epidemiolog-ical considerations. These results were in agreement with reports of Friedhoff (1997); Hashemi (1997); Walker and Koney (1999); Gholam (2001); Bell-Sakyi et al. (2004).

Examination of haemolymph and eggs-smears, of Hyalomma dromedarii ticks revealed the presence of five forms which were club, banana, spindle, crescentic and rod forms. In Sharkia Governorate in Egypt, Ahmed (1980) showed that one of the examined haemolymph specimens of 20 field collected $H$. ticks was positive for the developmental stages of Theileria species. Fahmy (1980) revealed that $65.2 \%$ of the collected $H$. males ticks and $59.5 \%$ of the females were infected with the developmental stages of Theileria species in Giza Governorate in Egypt. Also, El-Seify (1980); El Bahi (1986); Hamed et al. (2011) in Egypt observed the developmental forms of Piroplasma (Theileria) in haemolymph smears of $H$. spp. females which were club, banana and crescent shapes. These forms were observed by El Bahi (1986); Hamed et al. (2011). The developmental stages of Theileria spp., observed in the salivary glands of engorged nymph and moderate fed and unfed adult of Hyalomma dromedarii was the spindle shaped form Our results were similar with those obtained by El-Seify1 (980); Don et al. (1982); El Bahi (1986); Zapf and Schein (1994); Ulrich et al. (2007). 


\section{REFERENCES}

Abdel-Baki, S.M. (2001): Prevalence of external parasites in the south eastern desert of Egypt J. Egypt Soc. Parasitol., 31(1): 223-32.

Abunna, F.; Kasasa, D.; Shelima, B.; Megersa, B.; Regassa, A. and Amenu, K. (2009): Survey of tick infestation in small ruminants of Miesso district, West Harergie, Oromia Region, Ethiopia Trop. Anim. Health. Prod., 41(6): 969-72.

Ahmed, B.A.H. (1980): Some biological studies on blood parasites in farm animals. Ph.D.Thesis, Fac. Vet. Med. Zagazig Univ. 138p.

Ali, A. (2004): Studies on immune response of rabbits to Hylomma anatolicum anatolicum and Hylomma dromedarii (Acari: Ixodidae). Ph.D. thesis, Univ. of Khartoum.

Al-Khalifa, M.S.; Khalil, G.M.K. and Diab, F.M. (2007): A Two Years Study of Ticks infesting Goats and Sheep in Abha, Saudi Arabia. Journal of Biological Sciences 14 (1) 83-91.

Alsaad, K.M. (2009): Clinical hematological and biochemical studied of anaplasmosis in Arabian on humped camels (Camels dromedaries). J. animal Vet. Advances 8(11): 2160 -2109) ISSN: 1680-5593.

Barnett, S.F. (1974a): Economical aspects of protozoal tick-borne diseases in livestock in parts of the world other than Britain. Bull. Off. Int. Epiz. 81(1-2): 183-196.

Barnett, S.F. (1974b): Economical aspects of protozoal tick-borne disease control in Britain. Bull. Off. Int. Epiz. 81(1-2): 167-182.

Bell-Sakyi, L.; Koney, E.B.M.; Dogbey, O. and Walker, A.R. (2004): Incidence and prevalence of tick-borne haemoparasites in domestic ruminants in Ghana. Vet. Parasito, 124 (1-2): 25-42.

Burgdorfer, W. (1970): Haemolymph test A technique for detection of rickettsiae in ticks. Amer. J. Trop. Med. Hyg.19 (6): 1010-1014.

Caeiro, V. (1999): General review of ticks species present in Portugal Lab. De parasit. univer. De Evora. Portugal. Supp. 1-15.

Diab, F.M.; EL Kady, G.A and Shouky, A. (2001): Bionomics of ticks collected from Sinai. 2. Abundance, attachment sites and density of ticks infesting Arabian camels. Journal of Egyptian Society of Parasitology, 31: 479-489

Don, W.F.; Gottfried, B. and Stephen, D. (1982): Salivary gland of the tick vector of east coast fever. III. The ultrastructure of sporogony in Theileria parva. Inte Lab. Res. Anim Dis. 14(1): 183-206. 
El Bahi, N.A.M.A. (1986): Some Studies on Tick-Borne Diseases among Ruminants in Fayoum Governorate. M.Sc. Thesis, Fac. Vet.Med., Cairo Univ. 112p

El Kammah, K.M.; Oyoun, L.M.; El Kady, G.A. and Shafy, S.A. (2001): Investigation of blood parasites in livestock infested with argasid and ixodid ticks in Egypt. J. Egypt Soc Parasitol.; 31(2): 365-71.

El-Fayoumy, M.M.; Abou Elnga, T.R.; Abd El-Baky, S.M.M. and Abdou, T.A. (2005): Prevalence and of camel theileriosis and its vector tick in North Coast of Egypt. Journal of the Egyptian Veterinary Medical Association 65: 291-302.

El-Ghali, A. and Hassan, S.M. (2009): Ticks (Acari: Ixodidae) infesting camels (Camelus dromedarius) in Northern Sudan Onderstepoort J. Vet. Res., 76: 177-185.

El-Refaii M.A.; Wahba, A.A. and Gehan, J.S. (1998): Studies on theileria infection among slaughtered camels in Egypt J. Med. Sci. 19(1) $1-17$.

El-Refaii, M.A. and Wahba, A.A. (2003): Amblyomma lepidum Donitz, 1909 (Acari: Ixodidae) recovered from camels in Egypt. Egypt. J. Agric. Res., 81(1) 229-238

El-Seify, O.El.N.M. (1980): Studies on the role played by equines ticks in transmitting protozoal diseases. M. V.Sc. Cairo University.

Fahmy, M.M. (1980): Studies on cattle ticks and parasitic tick -borne diseases Giza. Governorate, M.V. sc. Thesis Fac. Vet. Med. Univ.

Friedhoff, K.T. (1997): Tick-borne diseases of sheep and goats caused by Babesia, Theileria or Anaplasma spp., Parassitologia 39, pp. 99-109.

Gerrit, $U$. (1986): Highlights in the recent research on tick-borne diseases of domestic animals.J. parassit.72 (4), pp. 485-491.

Gholam, R.R. (2001): An epidemiological study on ovine babesiosis in the mashhad suburb area,province of khorasan, Iran (an epidemiological study on ovine babesiosis in the mashhad suburb area, province of khorasan, Iran). Vet. Parasito. 108,(2): 109-115

Hadani, Y.A.; Galker, F. and Rosen, S. (1996): The seasonal occurrence of ticks (Acari: Ixodidae) on sheep and in the field in the Judean area of Israel. Exp. App. 20: 47-56.

Hamed, M.I.; Zitoun, A.M.A.; El-Allawy, T.A.A. and Mourad, I.M. (2011): Investigation of Theileria camelensis in camels infested by Hyalomma dromedarii ticks in Upper Egypt. Advanced Vet. Res. (1) 4-7. 
Hashemi, F.R. (1997): Tick-borne diseases of sheep and goats and their related vectors in Iran. Parassitologia, 39(2):115-7.

Higgins, A.T. (1984): The camel in health and disease, Balliere Tindall, London, Philadeliphia, Toronto.

Hoogstaal, H. (1956): African Ixodoidea. 1- Ticks of the Sudan (with special reference to equatorial province and with preliminary review of the genera Booplius, Margaropus and hyalomma). Dep.Navy, Bur. Med. Surg., Washington D.C. 1101 pp.

Hoogstraal, H. and Kaiser, M.N. (1959): Observations on Egyptian Hyalomma ticks (Ixodoidea: Ixodidae). 5. Biological notes and differences in identity of Hyalomma anatolicum and its subspecies anatolicum Koch and excavatum Koch among Russian and other workers identity of Hyalomma lusitanicum Koch. Entomological Society of America, 52: 243

Iqbal, M. (2005): Identification and chemotherapy of ectoparasites of camels (Camelus dromedarius) in Dera Ghazi.Khan UVASL Lahore (Pakistan), P. 66.

Kaiser, M..N.; Sutherst, R.W.; Bbourne, A.S.; Gorissen, L. and Floyd, R.B. (1988): Population dynamics of ticks on Ankole cattle in five ecological zones in Burundi and strategies for their control. Preventive Veterinary Medicine, 6: 199-222.

Karrar, G.; Kaiser, M.N. and Hoogstraal, H. (1963): Ecology and hostrelationship of ticks (Ixodoidea) infesting domestic animals in Kassala Province, Sudan with special reference to Amblyomma lepidum Donitz. Bulletin of Entomological Research, 54: 509-522.

Kruses, G.O.W. and Pritchard, M.H. (1982): The collection and preservation of animals parasites . Uni. Nebraska, Lincoln and London $141 \mathrm{pp}$.

Lawrence, R. and Thomas, G. (1987): Parasites: Aguide to laboratory procedures and identification (1st Ed). American society clinical pathologist, Chicago.

Levine, V.D. (1985): Veterinary protozoology 1st Iowa State Univ. Press Ames.

Mamak, N.; Gençer, L.; Ozkanlar, Y.E. and Ozçelik, S. (2006): Determination of tick species and treatment of cows, sheep and goats in the Sivas-Zara region. Turkiye Parazitol Derg. 30(3): 209-12.

Mazyad, S.A. and Khalaf, S.A. (2002): Studies on Theileria and Babesia infecting live and slaughtered animals in Al Arish and El Hasanah, 
North Sinai Governorate, Egypt. J. Egypt. Soc. Parasitol. 32: 601- 610.

Mohammad, Y. and Ali, H. (2006): Prevalence and ectoparasites fauna of sheep and goats flock in Uremia Suburb, Iran Veteranarski 76(5), 431-442.

Nassar, M.A. (1992): Theileria infection in camels (camelus dromedaries) in Egypt. Vet. Parasit. (43): 147-149.

Njanja, J.C.F.T.; Rinkanya, F.G.H. and Kiara, K. (1991): Ticks of camels, sheep and goats in Northwestern Kenya rangelands. I. J. of Pest Manag., 37(2) : 166 - 168.

Norval R.A.I.; Perry, B.D. and Young, A.S. (1992): The Epidemiology of Theileriosis in Africa, Academic Press, London. $481 \mathrm{pp}$.

Omer, L.T.M. Kadir, M.A.A.; Seitzer, U. and Ahmed, J.S. (2007): A Survey of ticks (Acari:Ixodidae) on cattle, sheep and goats in the Dohuk Governorate, Iraq. Parasitology Research. 101 (2) ISSN: 0932-0113.

Osman, A.M. (1997): Ticks infesting sheep and goats in the Sudan. Parassitologia; 39(2): 139-42.

Sajid, S.M.; Iqbal, Z.; Khan, M.N. and Muhammad, G. (2008): Point Prevalence of Hard Ticks (Ixodids) Infesting Domestic Ruminants of Lower Punjab, Pakistan. Inter. J. of Agriculture and Biology, 10: 349-351.

Sajid, S.M. (2007): Epidemiology and acaricidal resistance of tick population infesting domestic ruminants Ph.D. Thesis, Pakistan Univ.

Schein, E. and Friedhoff, K.T. (1978): Lichtmikroskopische Untersuchungen über die Entwicklung von Theileria annulata (Dschunkowsky and Luhs, 1904) in Hyalomma anatolilcum excavatum (Koch, 1844). II Die Entwicklung in Hämolymphe und Speicheldrusen. Z Parasitenkd 56: 287-303. Thesis, Fac. Vet. Med. Zagazig Univ. 138p.

Uilenberg, G. (1981): Theilerial species of domestic livestock In: A.D. Irvin, M.P. Cunningham and A.S. Young, Editors, Advances in the Control of Theileriosis, Martinus Nijhoff Publishers, The Hague. 4-37.

Ulrich, G.M.; Heinz, M.; Eberhard, S.; Wolf, P.V. and Karl, T.F. (2007): Ultrastructural Study on the Development of Babesia equi (Coccidia: Piroplasmia) in the Salivary Glands of its Vector Ticks. J. of Eukaryotic Microbiology Volume 30, Issue, DOI: 10.1111 . 
Van Straten, M. and Jongejan, F. (1993): Tick (Acari: Ixoidae) infesting the Arabian camel (Camels dromedaries) in the Sinai, Egypt with a note on the acaricidal efficacy. Experimental and Applied Acarology 17: 605-616.

Walker, A.R. and Koney, E.B.M. (1999): Distribution of ticks (Acari: Ixodida) infesting domestic ruminants in Ghana, Bull Ent. Res. 89, pp. 473-479.

Walker, A.R.; Bouatour, A.; Camicas, J.L.; Estrada Pena, A.; Horak, I.G.; Latif, A.A.; Pegram, R.G. and Preston, P.M. (2003): Ticks of domestic animals in Africa. A guide to identification of species. Bioscience report, 42 Comiston Drive, Edinburgh EH10 5 QR Scotland, UK.

Zapf, F. and Schein, E. (1994): New findings in the development of Babesia (Theileria) equi (Laveran, 1901) in the salivary glands of the vector ticks, Hyalomma species. Parasitol. Res.; 80(7):543-8.

Zeleke, M. and Bekele, T. (2004): Species of ticks and their seasonal population dynamics in eastern Ethiopia. Trop. An. Heal. and Prod., 36 (3): 225-231. 
Assiut Vet. Med. J. Vol. 57 No. 130 July 2011 
Assiut Vet. Med. J. Vol. 57 No. 130 July 2011 
Assiut Vet. Med. J. Vol. 57 No. 130 July 2011 
Assiut Vet. Med. J. Vol. 57 No. 130 July 2011 
Assiut Vet. Med. J. Vol. 57 No. 130 July 2011 
Assiut Vet. Med. J. Vol. 57 No. 130 July 2011 
Assiut Vet. Med. J. Vol. 57 No. 130 July 2011 
Assiut Vet. Med. J. Vol. 57 No. 130 July 2011 
Assiut Vet. Med. J. Vol. 57 No. 130 July 2011 